\title{
Slow Subcarrier-Hopped Space Division Multiple Access OFDM Systems
}

\author{
M. Jiang, S.X. Ng and L. Hanzo ${ }^{1}$ \\ School of ECS, Univ. of Southampton, SO17 1BJ, UK. \\ Tel: +44-703-593 125, Fax: +44-703-593 045 \\ Email: ${ }^{1}$ lh@ecs.soton.ac.uk, http://www-mobile.ecs.soton.ac.uk
}

\begin{abstract}
Recently Space Division Multiple Access (SDMA) assisted Multi-Input-Multi-Output (MIMO) OFDM systems invoking Multi-User Detection (MUD) techniques have attracted substantial research interests, which are capable of exploiting both transmitter multiplexing gain and receiver diversity gain. Furthermore, the classic Frequency-Hopping (FH) technique can be effectively amalgamated with SDMA-OFDM systems, resulting in Frequency-Hopped (FH) SDMA-OFDM. In this paper we devise a Turbo Trellis Coded Modulation (TTCM) assisted subcarrierbased FH/SDMA-OFDM scheme, which may be able to fully exploit the attainable frequency diversity, while exhibiting a high Multi-User-Interference (MUI) resistance. In the high-throughput scenario investigated, the proposed Uniform Slow-SubCarrierHopped (USSCH) SDMA-OFDM system was capable of achieving 6dB $E_{b} / N_{0}$ gain at the BER of $10^{-4}$ over the conventional SDMA-OFDM system, while maintaining a similar complexity.
\end{abstract}

\section{INTRODUCTION}

Spread-Spectrum Multiple Access (SSMA) schemes have been widely investigated, since they exhibit a range of attractive properties, including the ability of combating various types of interference. A classic SSMA scheme is constituted by Frequency-Hopped SSMA (FH/SSMA) [1], where the total available system bandwidth is divided into a number of sub-bands shared by a number of users. An appropriate number of subcarriers can be assigned to each of these subbands, which may experience different channel qualities and hence may deliver different type of services. This flexibility is attractive, when aiming for supporting future multimedia services, where variable bit rates associated with different Quality-of-Service (QoS) are required by different applications.

FH can be effectively amalgamated with the well-established technique of Orthogonal Frequency Division Multiplexing (OFDM) [2], which exhibits a number of advantages over more traditional multiplexing techniques, resulting in FH/OFDM systems [3]. Furthermore, Space Division Multiple Access (SDMA) based OFDM [2] communication invoking Multi-User Detection (MUD) [4] techniques has recently attracted intensive research interests. In SDMA Multi-InputMulti-Output (MIMO) systems the transmitted signals of $L$ simultaneous uplink mobile users - each equipped with a single transmit antenna - are received by the $P$ different receiver antennas of the Base Station (BS). At the BS the individual users' signals are separated with the aid of their unique, user-specific spatial signature constituted by their channel transfer functions or, equivalently, Channel Impulse Responses (CIRs). Based on combining both FH and SDMA-OFDM techniques, the concept of FH/SDMA-OFDM systems may be derived. In order to fully exploit the potential benefits of $\mathrm{FH}$, the em-

Acknowledgements: The financial support of the Mobile VCE is gratefully acknowledged. ployment of Forward Error Correction (FEC) schemes is necessary. In this paper, Turbo Trellis Coded Modulation (TTCM) [5] is considered, which is capable of achieving a substantial coding gain without bandwidth expansion, and was shown to generally provides the best performance among various Coded Modulation (CM) [5] schemes in the context of Minimum Mean-Square Error (MMSE) multi-user detected SDMA-OFDM systems [6].

Furthermore, the performance of the FH/SDMA-OFDM system may be significantly improved, if the proposed novel type of FH technique, referred to here as SubCarrier-Hopping $(\mathrm{SCH})$ is employed. The philosophy of the SCH technique is based on the concept of Orthogonal Frequency Division Multiple Access (OFDMA) [3]. More specifically, in this paper a subcarrier-based Slow Frequency-Hopping (SFH) technique is combined with a TTCM-aided SDMA-OFDM system, which may be referred to as Slow-SubCarrier-Hopping ( $\mathrm{SSCH}$ ) assisted TTCM-aided SDMA-OFDM, where each subcarrier is shared by a certain number of users in the context of the OFDM symbol's frequency-domain representation. It will be shown in our forthcoming discourse that with the aid of a carefully-designed Uniform SSCH (USSCH) pattern, the MUI experienced by the SDMA-OFDM system can be effectively suppressed, resulting in a significant performance improvement. Furthermore, the USSCH/SDMA-OFDM system is capable of significantly outperforming the conventional SDMAOFDM system [2] dispensing with the employment of hopping techniques, especially in high-throughput scenarios, while maintaining a similar complexity.

The structure of this paper is as follows. The SDMA MIMO channel model used is described in Section 2, while the detailed system description is provided in Section 3. The numerical results are presented in Section 4, followed by our complexity analysis in Section 5 . Our final conclusions are offered in Section 6.

\section{SDMA MIMO CHANNEL MODEL}

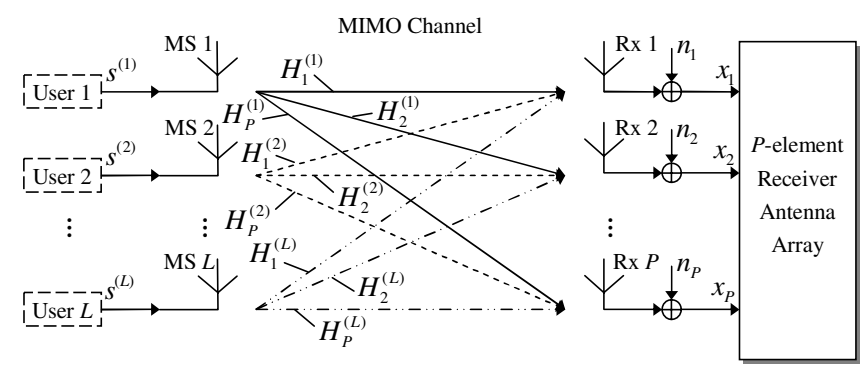

Figure 1: Schematic of the SDMA uplink MIMO channel model [2], where each of the $L$ mobile users has a single transmit antenna and the BS's receiver is assisted by a $P$-element antenna front-end. 
Figure 1 shows the SDMA uplink MIMO channel model, where each of the $L$ simultaneous mobile users employs a single transmit antenna at the mobile station (MS), while the BS's receiver exploits $P$ antennas. At the $k^{t h}$ subcarrier of the $n^{\text {th }}$ OFDM symbol received by the $P$-element receiver antenna array we have the received complex signal vector $\mathbf{x}[n, k]$, which is constituted by the superposition of the independently faded signals associated with the $L$ mobile users and contaminated by the Additive White Gaussian Noise (AWGN), expressed as:

$$
\mathbf{x}=\mathbf{H s}+\mathbf{n},
$$

where the $(P \times 1)$-dimensional vector $\mathbf{x}$, the $(L \times 1)$-dimensional vector $\mathbf{s}$ and the $(P \times 1)$-dimensional vector $\mathbf{n}$ are the received, transmitted and noise signals, respectively. Here we have omitted the indices $[n, k]$ for each vector for the sake of notational convenience. Specifically, the vectors $\mathbf{x}, \mathbf{s}$ and $\mathbf{n}$ are given by:

$$
\begin{aligned}
\mathbf{x} & =\left(x_{1}, x_{2}, \ldots, x_{P}\right)^{T}, \\
\mathbf{s} & =\left(s^{(1)}, s^{(2)}, \ldots, s^{(L)}\right)^{T}, \\
\mathbf{n} & =\left(n_{1}, n_{2}, \ldots, n_{P}\right)^{T} .
\end{aligned}
$$

The $(P \times L)$-dimensional matrix $\mathbf{H}$ contains the frequency-domain channel transfer functions (FD-CHTF) of the $L$ users and is given by:

$$
\mathbf{H}=\left(\mathbf{H}^{(1)}, \mathbf{H}^{(2)}, \ldots, \mathbf{H}^{(L)}\right),
$$

where $\mathbf{H}^{(l)}(l=1, \ldots, L)$ is the vector of the FD-CHTFs associated with the transmission paths from the $l^{t h}$ user's transmit antenna to each element of the $P$-element receiver antenna array, expressed as:

$$
\mathbf{H}^{(l)}=\left(H_{1}^{(l)}, H_{2}^{(l)}, \ldots, H_{P}^{(l)}\right)^{T}, \quad l=\{1, \ldots, L\},
$$

where $\mathbf{H}^{(l)}(l=1, \ldots, L)$ are independent between different users.

\section{SYSTEM MODEL}

\subsection{System Overview}

In conventional SFH-assisted OFDM systems the total available system bandwidth $W$ is divided into a number of sub-bands denoted by $q=1, \ldots, Q$. Each sub-band is assigned a carrier frequency $f_{q}$, which is used for carrying the OFDM signal to be transmitted in this sub-band, when it is activated. Furthermore, each of the $Q$ sub-bands has $K$ number of subcarriers, each having a subcarrier bandwidth of $W_{s c}$, and we have $W=W_{s c} \times K \times Q$. In the context of the SFH/SDMA-OFDM system, during a FH dwell time $T_{h}$, each of the $L$ simultaneous users supported is assigned one of the $Q$ sub-bands associated with a specific carrier frequency $f_{q}$, which is activated by a frequency synthesizer according to the user-specific SFH pattern used. The symbol duration $T_{s}$ obeys $\beta_{S}=T_{h} / T_{s}>1$, where $\beta_{S}$ is an integer, implying SFH. Using SFH makes it feasible to use coherent demodulation at the receiver side, since the hopping rate is slower than the data rate.

By contrast, in the proposed SSCH/SDMA-OFDM system a subcarrier based, rather than sub-band based SFH technique is invoked. More specifically, in SSCH systems the same system bandwidth $W$ is partitioned into $Q=W / W_{s c}$ number of subcarriers. While a SFH user exploits all the $K$ subcarriers of a SFH sub-band during the duration of $T_{h}$, a SSCH user employing the same number of $K$ subcarriers can potentially select any $K$ of the $Q$ available subcarriers, where $0<K<Q$, without decreasing the throughput. Furthermore, in $\mathrm{SSCH}$ systems a high MUI is expected to contaminate the dispersed subcarriers activated by a number of users, which is different from the situation experienced in the conventional SFH scenario, where potentially all the consecutive subcarriers of a sub-band hosting a number of users may be at a risk of being severely corrupted. Moreover, the SSCH scheme is capable of more efficiently exploiting the benefits of frequency diversity in comparison to the conventional SFH arrangement, since a deep fade which may corrupt many consecutive subcarriers of a SFH user, may only affect a small fraction of the subcarriers used by a SSCH user, as an added bonus of the employment of discontinuous subcarriers.

\subsection{Transmitter and Receiver Architecture}
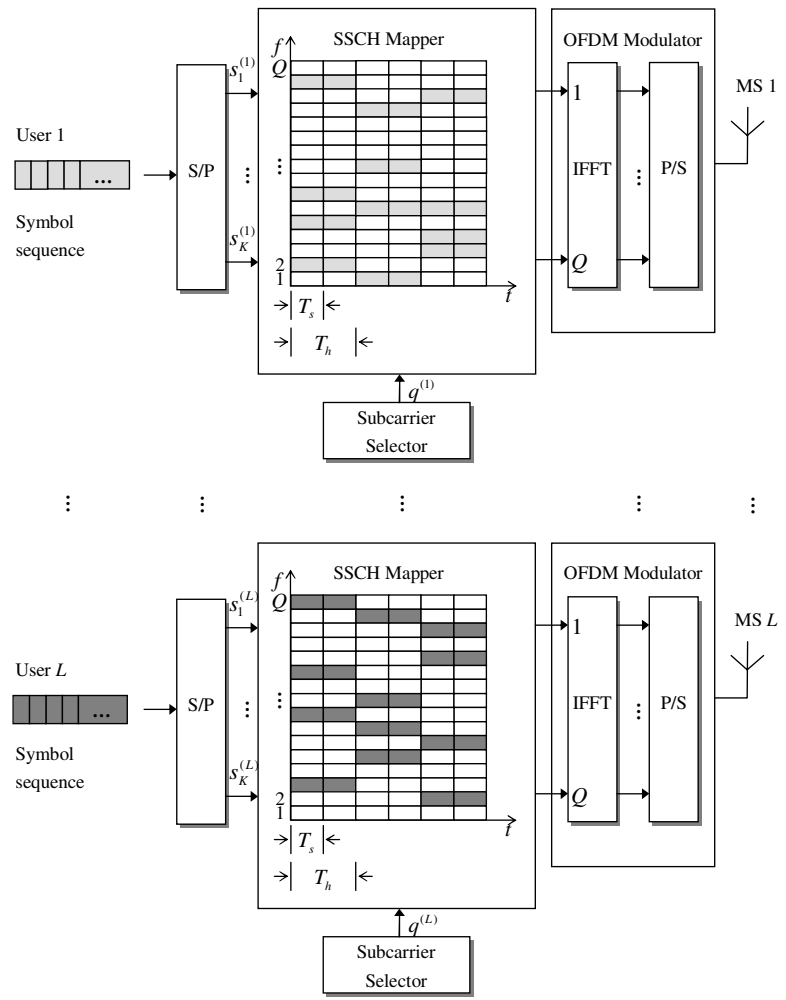

Figure 2: Illustration of the $L$-users' SSCH/SDMA-OFDM transmitters. As an example, random SSCH patterns are used.

The transmitter structure of the TTCM-assisted SSCH/SDMAOFDM system is portrayed in Figure 2. As seen in Figure 2, the TTCM-coded symbols are first S/P-converted and forwarded to the $\mathrm{SSCH}$ mapper. More specifically, the $K$ number of information symbols of a user are mapped to $K$ out of $Q \mathrm{SSCH}$ subcarriers, where the activation strategy of the set of $K$ subcarriers depends on the specific $\mathrm{SSCH}$ algorithm used. For example, if pseudo-random hopping is employed, each user can independently select $K$ subcarriers according to the action of a pseudo-random subcarrier selector, as illustrated in Figure 2. Therefore, an ON-OFF type signaling scheme may be invoked, where the activated and deactivated status of specific subcarriers represent the $\mathrm{ON}$ and $\mathrm{OFF}$ states, respectively. Then the total number of $Q$ subcarriers can be processed by a $Q$-point Inverse Fast Fourier Transform (IFFT). Since the deactivated subcarriers deliver no information, the transmit power of each SSCH user is the same as that of a SFH user, if the same number of $K$ activated subcarriers 
are employed by both of them. After the IFFT-based OFDM modulation, the OFDM symbols will be transmitted by the MS to the SDMA MIMO channel of Figure 1 using a specific carrier frequency $f_{c}$.

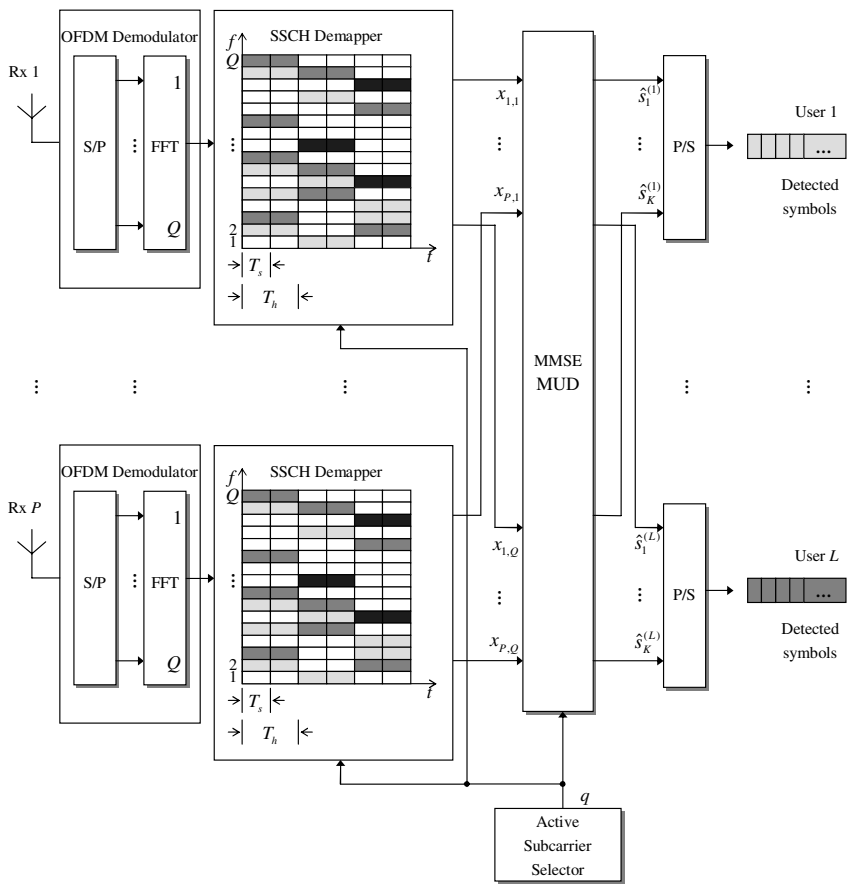

Figure 3: Illustration of the $L$-users' SSCH/SDMA-OFDM receivers. As an example, random $\mathrm{SSCH}$ patterns are used.

The receiver structure of the SSCH/SDMA-OFDM system follows the inverse of the $\mathrm{SSCH}$ transmitter structure, as portrayed in Figure 3. First Fast Fourier Transform (FFT) based OFDM demodulation is invoked, followed by the SSCH demapping. Given the knowledge of the SSCH pattern, the BS's active subcarrier selector simultaneously selects the active subcarriers at the $P$ receivers, and the resultant composite multi-user output signal is forwarded to the MUD for multi-user detection. As an example, the MMSE MUD is pictured in Figure 3, but other MUDs are equally applicable. The separated different users' signals are then P/S-converted and forwarded to the user-specific TTCM channel decoders.

\subsection{Subcarrier-Hopping Design}

In this paper, two types of hopping patterns are considered. We refer to the first scheme as the Random SSCH (RSSCH) pattern, where each user independently selects any $K$ out of the total of $Q$ available subcarriers during the period of $T_{h}$. In RSSCH-based systems, the subcarriers activated by a high number of users will suffer an excessive MUI, resulting in an increased average Bit Error Ratio (BER). By contrast, in the second SSCH strategy considered, all users' hopping patterns are jointly designed so that the number of interfering users at each subcarrier is as similar as possible, which minimizes the average MUI. We refer to this strategy as the Uniform SSCH (USSCH) scheme. In the proposed USSCH scheme, the $Q$ available subcarriers are first partitioned into $K$ consecutive subcarrier groups, each of which has $Q_{g}=Q / K$ number of subcarriers. Then an $L$-iteration USSCH pattern assignment algorithm is invoked for each of the $K$ groups, namely from the first to the $K^{t h}$ group, for the sake of gener- ating a specific length- $K$ pattern set for each of the $L$ users.

More specifically, the algorithm invoked in the $k^{t h}$ subcarrier group is described as follows. Initially a remaining user set $\mathbf{A}^{(1)}$ containing all the $L$ users is created. During the $l^{t h}(l=1, \ldots, L)$ iteration, a subcarrier index $b_{k}^{(l)}$ is generated by calculating $l \bmod Q_{g}$, where $\bmod$ represents the modulo operation, and a user $u^{(l)}$ is randomly selected from $\mathbf{A}^{(l)}$ based on the uniform probability of:

$$
p^{(l)}=1 /(L-l+1) .
$$

Then the $\left(b_{k}^{(l)}\right)^{t h}$ subcarrier within this specific group will be assigned to user $u^{(l)}$, and $\mathbf{A}^{(l)}$ is updated by removing user $u^{(l)}$ from it, resulting in $\mathbf{A}^{(l+1)}$, which contains the remaining $(L-l)$ number of users. Then, the algorithm proceeds to the next, i.e. to the $(l+1)^{s t}$ iteration, and this iterative subcarrier to user assignment process continues, until the $L^{t h}$ iteration is reached. By this time each of the $L$ users has been assigned a subcarrier of the $k^{\text {th }}$ group. Hence, a vector $\mathbf{b}_{k}=\left[b_{k}^{(1)}, b_{k}^{(2)}, \ldots, b_{k}^{(L)}\right]$ is generated, where $b_{k}^{(l)}(l=1, \ldots, L)$ constitutes the $k^{t h}$ subcarrier of the $l^{t h}$ user's length- $K$ USSCH pattern set $^{1}$. Then the vector-generating routine described above is invoked for the next, i.e. for the $(k+1)^{s t}$ subcarrier group. When all the $K$ subcarrier groups have been processed, a set containing $K$ number of length- $L$ vectors $\mathbf{b}_{k}(k=1, \ldots, K)$ has been generated, explicitly indicating, which specific subcarrier of group $k$ has been assigned to which of the $L$ users. Viewing this subcarrier allocation from the users' perspective, the $l^{\text {th }}$ user's specific length- $K$ USSCH pattern set $\mathfrak{b}^{(l)}$ is created by choosing that specific subcarrier corresponding to the $l^{\text {th }}$ element of each of the $K$ vectors, which has been assigned to user $l$, resulting in $\mathfrak{b}^{(l)}=\left[b_{1}^{(l)}, b_{2}^{(l)}, \ldots, b_{K}^{(l)}\right], l \in\{1, \ldots, L\}$.

Note that the above subcarrier group based algorithm employs the full permutation theory [7]. More explicitly, each of the $K$ number of length- $L$ vectors $\mathbf{b}_{k}(k=1, \ldots, K)$ associated with the $k^{t h}$ subcarrier group is an element of the $L$-order full permutation set [7], and is generated at a constant probability of:

$$
p=\prod_{l=1}^{L} p^{(l)}=\frac{1}{L !},
$$

where $(\cdot)$ ! represents the factorial operation. More specifically, given a total number of $L$ users, the associated $L$-order full permutation set can be expressed as:

$$
\mathbf{B}_{L}=\left\{\mathbf{b}_{1}, \mathbf{b}_{2}, \ldots, \mathbf{b}_{L !}\right\}
$$

where the element vectors are given by $\mathbf{b}_{k}=\left[b_{k}^{(1)}, b_{k}^{(2)}, \ldots, b_{k}^{(L)}\right](k=$ $1, \ldots, L$ !). Thus, the employment of the USSCH algorithm described above is conceptually equivalent to invoking the algorithm, which randomly selects one element vector from $\mathbf{B}_{L}$ of Equation 9 for each of the $K$ subcarrier groups, based on the uniform probability given by Equation 8. Therefore, this particular characteristic of the proposed USSCH pattern assignment algorithm may ensure that all users become capable of efficiently exploiting the maximum achievable frequency diversity offered by the system, since each user's specific length- $K$ USSCH pattern set is created by assigning a subcarrier from each of the $K$ groups across the entire system bandwidth in a "uniformrandom" manner. This also increases the chances of correcting the errors encountered at the uniformly-distributed corrupted subcarriers with the aid of the TTCM decoder.

Note that in the context of the uplink SDMA-OFDM systems employing RSSCH patterns, the MSs have to signal the patterns to the

${ }^{1}$ Recall that each user activates $K$ subcarriers for transmission and thus has to be assigned a length- $K$ USSCH pattern set. 
BS, so that appropriate dehopping operation can be invoked. A better design alternative might be for the BS to allocate the hopping patterns, but in this case it would be more natural to employ the USSCH scheme. More specifically, in the context of the USSCH scheme, all the USSCH patterns can be jointly allocated by the BS and be signalled to the MSs. In both scenarios, the signalling of the SSCH patterns will occupy part of the system's bandwidth, and hence decreasing the effective information data rate. If explicit knowledge of the hopping patterns is not available, the blind joint FH pattern and data soft-detection techniques of [1] may be invoked, which was shown to be capable of approaching the BER performance of the system using explicit FH pattern knowledge at the cost of an increased complexity.

\subsection{Multi-User Detection}

As mentioned earlier, MUD schemes are invoked at the receiver of the SDMA-OFDM system for the sake of detecting the received signals of different users. Recently, a novel MMSE-assisted Iterative Genetic Algorithm (IGA) based (MMSE-IGA) MUD [8] has been proposed for employment in SDMA-OFDM systems, which is capable of achieving a similar performance to that attained by the optimum Maximum Likelihood (ML) MUD [2] at a significantly lower complexity, especially in high-throughput scenarios. Furthermore, the concatenated MMSE-IGA MUD of [8] may also be employed in the proposed SSCH/SDMA-OFDM system. More specifically, at the $q^{t h}$ active subcarrier, the initial estimated signal vector $\hat{\mathrm{S}}_{\mathrm{MMSE}_{q}} \in \mathbb{C}^{L_{q} \times 1}$ generated from the transmitted signal $\mathbf{s}_{q}$ of the $L_{q}\left(1 \leq L_{q} \leq L\right)$ simultaneous users that activate this specific subcarrier, is obtained by linearly combining the received signal vector $\mathbf{x}_{q}$ with the aid of the MMSE MUD's array weight matrix, as follows:

$$
\hat{\mathbf{s}}_{\mathrm{MMSE}_{q}}=\mathbf{W}_{\mathrm{MMSE}_{q}}^{H} \mathbf{x}_{q},
$$

where the superscript $(\cdot)^{H}$ denotes the Hermitian transpose, and the MMSE-based weight matrix $\mathbf{W}_{\text {MMSE }_{q}} \in \mathbb{C}^{P \times L_{q}}$ is given by [2]:

$$
\mathbf{W}_{\text {MMSE }_{q}}=\left(\mathbf{H}_{q} \mathbf{H}_{q}^{H}+\sigma_{n}^{2} \mathbf{I}\right)^{-1} \mathbf{H}_{q},
$$

while $\mathbf{I}$ is the identity matrix and $\sigma_{n}^{2}$ is the AWGN noise variance. Once the MMSE-based detection is completed, the resultant output can be forwarded to the concatenated IGA MUD [8] for the secondstage iterative detection. More precisely, the IGA MUD evaluates a decision metric associated with the $P$ receivers, which is derived from the optimum ML-based decision metric [2], in order to detect the estimated transmitted symbol vector $\hat{\mathbf{s}}_{\mathrm{GA}_{q}}$. The decision metric required for the $p^{\text {th }}$ receiver antenna is defined by [8]:

$$
\Omega_{p, q}(\mathbf{s})=\left|x_{p, q}-\mathbf{H}_{p, q} \mathbf{s}\right|^{2},
$$

and $x_{p, q}$ is the received symbol at the $q^{t h}$ active subcarrier of the $p^{\text {th }}$ receiver, while $\mathbf{H}_{p, q}$ is the $p^{t h}$ row of the $\left(P \times L_{q}\right)$-dimensional channel transfer function matrix $\mathbf{H}_{q}$ related to the same subcarrier. Therefore the decision rule for the optimum multiuser detector associated with the $p^{t h}$ antenna is to choose that specific length- $L_{q}$ symbol vector $\mathbf{s}$, which minimizes the metric given in Equation 12. Since there are $P$ number of receivers, the combined joint metric is invoked [8]:

$$
\Omega_{q}(\mathbf{s})=\sum_{p=1}^{P} \Omega_{p, q}(\mathbf{s}) .
$$

Hence, the joint decision rule is to find that specific estimated transmitted $L_{q}$-symbol vector $\hat{\mathbf{s}}_{\mathrm{GA}_{q}}$, which minimizes $\Omega_{q}(\mathbf{s})$ in Equation 13 for the $q^{\text {th }}$ active subcarrier considered, which is formulated as:

$$
\hat{\mathbf{s}}_{\mathrm{GA}_{q}}=\arg \left\{\min _{\mathbf{s}}\left[\Omega_{q}(\mathbf{s})\right]\right\} .
$$

\section{SIMULATION RESULTS}

In this section, we characterize the performance of the TTCM-assisted SSCH/SDMA-OFDM system using the MMSE-IGA MUD of [8]. The simulation results were obtained using a 4QAM scheme communicating over the 3-path Short Wireless Asynchronous Transfer Mode (SWATM) CIR given on page 78 of [2], assuming that the channels' transfer functions are perfectly known. Each of the paths experiences independent Rayleigh fading having the same normalized Doppler frequencies of $f_{d}^{\prime}=1.235 \times 10^{-5}$. In the SFH-assisted systems a Random SFH (RSFH) pattern was used, while in the SSCHassisted systems the RSSCH and USSCH patterns were used. It was assumed in all FH-based schemes that the BS has a perfect knowledge of the hopping patterns. Recall that in RSFH/RSSCH/USSCH aided SDMA-OFDM systems the number of activated subcarriers $K$ is lower than that of the conventional SDMA-OFDM system [2], where all the users employ all the $Q$ number of available subcarriers for communication. For the sake of fair comparisons, the total system bandwidth was fixed and the number of users $L$ supported by the various FH-aided systems was increased, so that the same total throughout of $B_{\mathrm{T}}$ was maintained during an OFDM symbol, which is calculated by:

$$
B_{\mathrm{T}}=K \cdot L \cdot B P S[\text { bits }],
$$

where $B P S$ represents the number of Bits Per Symbol per subcarrier. When 4QAM is employed, we have $B P S=2$.

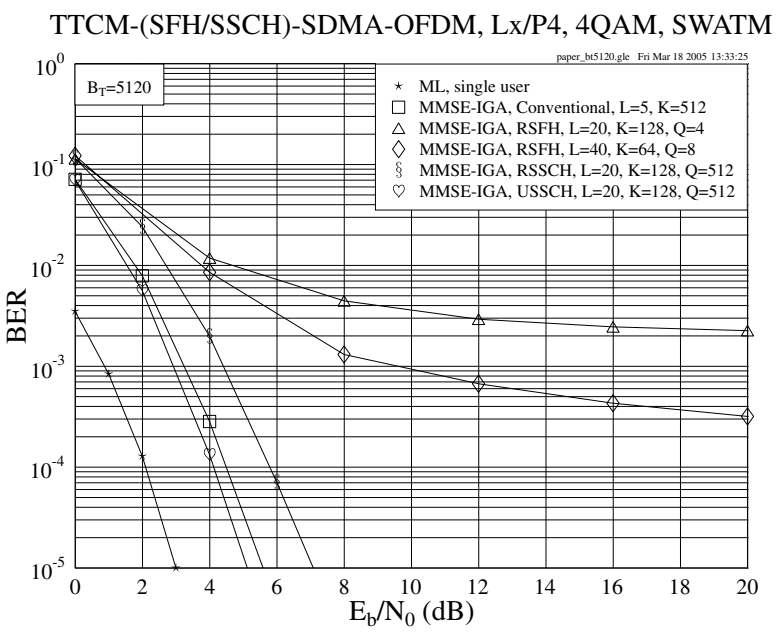

Figure 4: BER versus $E_{b} / N_{0}$ performance of the TTCMassisted conventional SDMA-OFDM and TTCM-assisted RSFH/RSSCH/USSCH aided SDMA-OFDM systems, where $L$ number of users were supported with the aid of $P=4$ receiver antenna elements. The associated overall system throughput was $\mathrm{B}_{\mathrm{T}}=\mathbf{5 1 2 0}$ bits

Figure 4 compares the BER performance of the various TTCMassisted RSFH/RSSCH/USSCH aided SDMA-OFDM systems and that of the TTCM-assisted conventional SDMA-OFDM system, where the MMSE-IGA MUD was employed. The performance of the singleuser SDMA-OFDM system employing the optimum ML MUD is also provided for reference. The same total throughout of $B_{\mathrm{T}}=5120$ bits was maintained for all schemes, except for the single-user arrangement. It is observed from Figure 4 that the RSFH-aided schemes suffered from an excessive MUI and high error floors were observed. By contrast, the random and uniform SSCH-assisted arrangements were capable of achieving a significantly better performance than their RSFH- 
based counterparts. Furthermore, the USSCH/SDMA-OFDM system slightly outperformed the conventional SDMA-OFDM system, while the RSSCH-aided system had an $E_{b} / N_{0}$ loss of about $2 \mathrm{~dB}$ at the BER of $10^{-5}$. This is because when RSSCH is invoked, some subcarriers may be assigned to an excessive number of users, resulting in a number of "MUI peaks" at the disadvantaged subcarriers, and thus degrading the system's overall average performance.

TTCM-(SFH/SSCH)-SDMA-OFDM, Lx/P4, 4QAM, SWATM

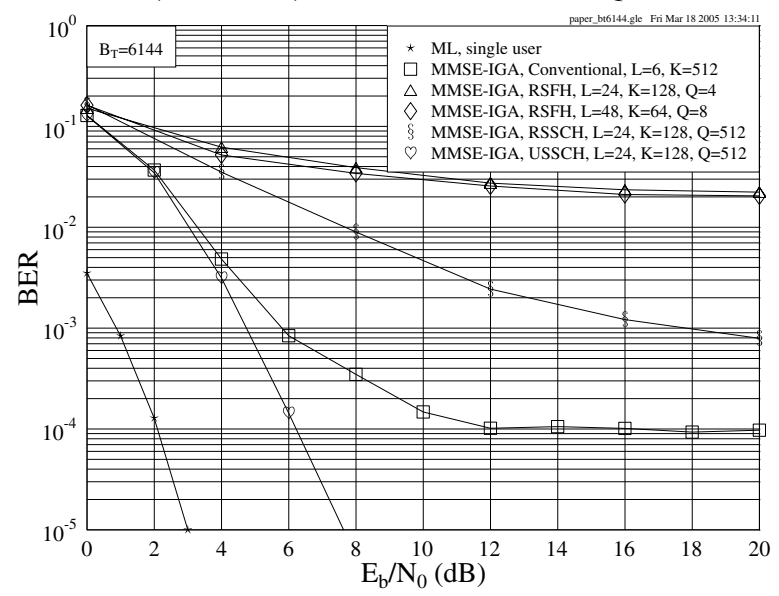

Figure 5: BER versus $E_{b} / N_{0}$ performance of the TTCMassisted conventional SDMA-OFDM and TTCM-assisted RSFH/RSSCH/USSCH aided SDMA-OFDM systems, where $L$ number of users were supported with the aid of $P=4$ receiver antenna elements. The associated overall system throughput was $\mathrm{B}_{\mathrm{T}}=\mathbf{6 1 4 4}$ bits.

Furthermore, when the total system throughput was further increased, for example in a scenario associated with $B_{\mathrm{T}}=6144$ bits, the USSCH/SDMA-OFDM system was capable of achieving a significantly better performance in comparison to the other systems, as shown in Figure 5. More precisely, the conventional and the RSFH or RSSCH aided schemes severely suffered from the excessive MUI and thus exhibited the corresponding error floors. By contrast, the USSCH arrangement successfully suppressed the MUI and eliminated the associated error floor, as seen in Figure 5. This significant BER performance improvement was achieved as a benefit of the USSCH strategy presented in Section 3.3, which effectively exploited the frequency diversity by dispersing the users' signals across the entire system bandwidth in a uniform-random manner. Hence, the TTCM decoder was capable of successfully correcting most of the near-uniformly scattered errors inherent in each user's transmitted signal, resulting in a significant performance improvement. For example, at an $E_{b} / N_{0}$ value of $8 \mathrm{~dB}$, the proposed USSCH/SDMA-OFDM system reduced the BER by about two, three and four magnitudes in comparison to the conventional SDMA-OFDM, the RSSCH/SDMA-OFDM and the RSFH/SDMA-OFDM systems, respectively, as shown in Figure 5.

\section{COMPLEXITY ANALYSIS}

As mentioned in Section 3.2, the Q-point IFFT employed by the SSCH system will inevitably impose a higher computational complexity than its SFH counterpart, where a $K$-point IFFT is used, regardless whether the RSSCH or USSCH scheme is employed, since we have $K<Q$. However, it has been demonstrated in Section 4 that SSCH systems typically achieve a significant performance improvement over $\mathrm{SFH}$ arrangements, hence their additional complexity cost is justified. Furthermore, in the context of SSCH systems the OFDM symbols modulate a single carrier frequency $f_{c}$, hence the frequency synthesizers necessary for both the SFH transmitter and receiver are not required, which simplifies the hardware implementation of the SSCH system.

On the other hand, concerning the RSSCH and the USSCH systems, a similar SSCH transmitter invoking a $Q$-point IFFT is employed, thus the transmitter's complexity is similar in both systems. However, the RSSCH arrangement imposes a potentially higher complexity at the receiver side. This is because the number of active users at each subcarrier is a random variable spanning from 1 to $L$, and hence a MUD that is capable of detecting a variable number of active users has to be employed. Naturally, this problem exists in all random FH/SCH based SDMA-OFDM systems. By contrast, a USSCH receiver has a complexity similar to that of conventional SDMA-OFDM systems, which employ an MUD designed for a fixed number of active users, since the USSCH users are uniformly distributed across the system's bandwidth.

\section{CONCLUSIONS}

From the investigations conducted, we conclude that the proposed USSCH/SDMA-OFDM system is capable of significantly outperforming the RSSCH/SDMA-OFDM, the SFH/SDMA-OFDM and the conventional SDMA-OFDM systems, when TTCM is employed. Furthermore, the USSCH-aided system exhibits a high resistance to the MUI incurred in high-throughput scenarios, and this superior performance is achieved at a similar computational complexity to that imposed by the conventional SDMA-OFDM arrangement.

\section{REFERENCES}

[1] L. Hanzo, L.-L. Yang, E.-L. Kuan, and K. Yen, Single- and MultiCarrier DS-CDMA: Multi-User Detection, Space-Time Spreading, Synchronisation and Standards. IEEE Press - John Wiley \& Sons Ltd., 2003.

[2] L. Hanzo, M. Münster, B. J. Choi, and T. Keller, OFDM and MCCDMA for Broadband Multi-user Communications, WLANs and Broadcasting. IEEE Press - John Wiley \& Sons Ltd., 2003.

[3] I. Koffman and V. Roman, "Broadband Wireless Access Solutions Based on OFDM Access in IEEE 802.16," IEEE Communications Magazine, vol. 40, pp. 96-103, April 2002.

[4] S. Verdu, Multiuser Detection. Cambridge University Press, 1998.

[5] L. Hanzo, T. H. Liew, and B. L. Yeap, Turbo Coding, Turbo Equalisation and Space-Time Coding for Transmission Over Fading Channels. New York, USA: IEEE Press - John Wiley \& Sons Ltd., 2002.

[6] M. Jiang, S. X. Ng, and L. Hanzo, "TCM, TTCM, BICM and BICM-ID Assisted MMSE Multi-User Detected SDMA-OFDM Using Walsh-Hadamard Spreading," in Proceedings of IEEE Vehicular Technology Conference '04 Spring, (Milan, Italy), pp. 1129-1133, May 17-19 2004.

[7] S. Lipschutz and M. L. Lipson, Schaum's Outline of Theory and Problems of Probability. Schaum's Outline Series, USA: McGraw-Hill Inc., second ed., 2000.

[8] M. Jiang, S. X. Ng, and L. Hanzo, "Hybrid Multi-User Detection for Turbo Trellis Coded Space Division Multiple Access OFDM Systems," submitted to IEEE Transactions on Vehicular Technology, November 2004. 